\title{
Enhanced carotid body chemosensory activity and the cardiovascular alterations induced by intermittent hypoxia
}

\author{
Rodrigo Iturriaga ${ }^{1 *}$, David C. Andrade ${ }^{1}$ and Rodrigo Del Rio ${ }^{2}$ \\ 'Laboratorio de Neurobiología, Departamento de Fisiología, Facultad de Ciencias Biológicas, Pontificia Universidad Católica de Chile, Santiago, Chile \\ 2 Laboratory of Cardiorespiratory Control, Center of Biomedical Research, Universidad Autónoma de Chile, Santiago, Chile
}

\author{
Edited by: \\ Harold D. Schultz, University of \\ Nebraska Medical Center, USA \\ Reviewed by: \\ Lucilia Neves Diogo, Universidade \\ Nova de Lisboa, Portugal \\ Barbara Morgan, University of \\ Wisconsin, USA \\ *Correspondence: \\ Rodrigo Iturriaga, Laboratorio de \\ Neurobiología, Facultad de Ciencias \\ Biológicas, Pontificia Universidad \\ Católica de Chile, Alameda 340, \\ Santiago 8320000, Chile \\ e-mail: riturriaga@bio.puc.cl
}

\begin{abstract}
The carotid body (CB) plays a main role in the maintenance of the oxygen homeostasis. The hypoxic stimulation of the CB increases the chemosensory discharge, which in turn elicits reflex sympathetic, cardiovascular, and ventilatory adjustments. An exacerbate carotid chemosensory activity has been associated with human sympathetic-mediated diseases such as hypertension, insulin resistance, heart failure, and obstructive sleep apnea (OSA). Indeed, the CB chemosensory discharge becomes tonically hypereactive in experimental models of OSA and heart failure. Chronic intermittent hypoxia $(\mathrm{ClH})$, a main feature of OSA, enhances CB chemosensory baseline discharges in normoxia and in response to hypoxia, inducing sympathetic overactivity and hypertension. Oxidative stress, increased levels of ET-1, Angiotensin II and pro-inflammatory cytokines, along with a reduced production of $\mathrm{NO}$ in the $\mathrm{CB}$, have been associated with the enhanced carotid chemosensory activity. In this review, we will discuss new evidence supporting a main role for the $\mathrm{CB}$ chemoreceptor in the autonomic and cardiorespiratory alterations induced by intermittent hypoxia, as well as the molecular mechanisms involved in the CB chemosensory potentiation.
\end{abstract}

Keywords: autonomic dysfunction, carotid body, intermittent hypoxia, hypertension, oxidative stress

\section{INTRODUCTION}

The carotid body (CB) located in the bifurcation of the carotid arteries is the main peripheral chemoreceptor sensing arterial levels of $\mathrm{PO}_{2}, \mathrm{PCO}_{2}$, and pH. Also, changes in blood flow, temperature, osmolarity and glucose are able to elicit $\mathrm{CB}$ chemosensory excitation (Gonzalez et al., 1994; Pardal and López-Barneo, 2002; Iturriaga and Alcayaga, 2004; Iturriaga et al., 2007). The CB consists of clusters of chemoreceptor (glomus or type I) cells organized around the capillary network, synaptically connected to the nerve terminals of sensory neurons whose somata are in the petrosal ganglion, and surrounded by sustentacular glial (type II) cells. The most accepted model for chemoreception proposes that hypoxia closes $\mathrm{K}^{+}$channels, leading to glomus cell depolarization, entry of $\mathrm{Ca}^{2+}$ and the release of excitatory transmitters (ACh and ATP), which in turn increases the discharge in the nerve endings of the chemosensory neurons (Iturriaga and Alcayaga, 2004; Iturriaga et al., 2007). In the last years, new exciting evidences have shown that the $\mathrm{CB}$ plays a crucial role in the pathogenesis of several human sympathetic-mediated diseases, including obstructive sleep apnea (OSA), congestive heart failure, resistant hypertension and insulin resistance (Koyama et al., 2000; Prabhakar et al., 2005; Schultz et al., 2007; Iturriaga et al., 2009; Abdala et al., 2012; Del Rio et al., 2013; Paton et al., 2013; Porzionato et al., 2013; Ribeiro et al., 2013). Accordingly, targeting the $\mathrm{CB}$ in several pathological conditions has been proposed to be a future promising therapeutic tool for the treatment of sympathetic-mediated diseases. Indeed, the selective ablation of the $\mathrm{CB}$ markedly improve rat survival in experimental heart failure (Del Rio et al., 2013), prevent the development of insulin resistance and hypertension in rats fed with high sucrose diet (Ribeiro et al., 2013) and reduced high blood pressure in neurogenic and resistant hypertension (McBryde et al., 2013; Paton et al., 2013).

\section{OBSTRUCTIVE SLEEP APNEA IS AN INDEPENDENT RISK FACTOR FOR SYSTEMIC HYPERTENSION}

The OSA syndrome elicited by repeated upper airways occlusion, is usually associated with daytime sleepiness, fatigue, and deficits in attention and executive function (Beebe and Gozal, 2002; Idiaquez et al., 2014). Furthermore, OSA is recognized as an independent risk factor for systemic hypertension ( $\sim 50 \%$ of OSA patients develop diurnal hypertension, Somers et al., 2008; Calhoun, 2010), and is associated with stroke, pulmonary hypertension, coronary artery disease and atrial fibrillation (Fletcher, 2000; Parati et al., 2007; Somers et al., 2008; Dempsey et al., 2010). Indeed, several epidemiological studies have shown that OSA is an independent risk factor for the progression of the hypertension, showing a positive relationship between the apnea/hypopnea index (AHI) and high blood pressures (Young et al., 1993; Peppard et al., 2000; Eckert and Malhotra, 2008; Marin et al., 2012). Moreover, results obtained from the Wisconsin Sleep Cohort (an ongoing 21-years longitudinal study performed on 1500 Wisconsin state employees) showed that untreated OSA patients have a high mortality risk associated with AHI (Nieto et al., 2000; Young et al., 2008). According to the "Recommendations for the management of 
patients with obstructive sleep apnoea and hypertension" recently published by the European Union Cooperation in Scientific and Technological Research Action B26 on OSA, with the endorsement of the European Respiratory Society and the European Society of Hypertension (Parati et al., 2013) OSA is defined as "The combination of at least five obstructive breathing episodes per hour during sleep (apnoea, hypopnoea and respiratory effort related arousal events) and the following diagnostic criteria (A and/or B to be fulfilled). A: Excessive daytime sleepiness that is not better explained by other factors. B: Two or more of the following symptoms that are not better explained by other factors: Choking or gasping during sleep, recurrent awakenings from sleep, unrefreshing sleep, daytime fatigue and impaired concentration." According to this study, the AHI defines the severity of OSA: mild OSA: AHI 5-15 events/h; moderate OSA: AHI 15-30 events/h and severe OSA: AHI > 30 events/h (Parati et al., 2013).

\section{PATHOPHYSIOLOGICAL MECHANISMS OF OSA-INDUCED HYPERTENSION}

The cyclic obstruction of the upper airways during OSA leads to intermittent hypoxia and hypercapnia, negative intrathoraxic pressure, sleep fragmentation, and micro-arousals (Somers et al., 2008; Dempsey et al., 2010). During the airway occlusion, the resulting hypoxia and hypercapnia stimulates the CB chemoreceptor eliciting reflex acute sympathetic, hypertensive and hyperventilatory responses (Gozal and KheirandishGozal, 2008; Somers et al., 2008; Garvey et al., 2009; Dempsey et al., 2010). Among these disturbances, the chronic intermittent hypoxia $(\mathrm{CIH})$ is considered the main factor for the development of diurnal hypertension (Lavie, 2003; Gozal and KheirandishGozal, 2008; Lévy et al., 2008; Somers et al., 2008; Arnardottir et al., 2009; Dempsey et al., 2010). Although the link between OSA and hypertension is well proved, the mechanisms underlying the pathogenesis of the hypertension are not entirely known. The most accepted proposal states that $\mathrm{CIH}$ elicits systemic oxidative stress, inflammation, and sympathetic hyperactivity, which led to endothelial dysfunction and the hypertension (Lavie, 2003; Somers et al., 2008; Garvey et al., 2009; Ryan et al., 2009; Dempsey et al., 2010). Nevertheless, conclusions from studies performed in OSA patients are controversial, because OSA patients often present concomitant morbidities (i.e., obesity and metabolic alterations), which are confounding factors that increase the cardiovascular risk. Thus, animal model of $\mathrm{CIH}$, which simulates the hypoxic-reoxygenation episodes and reproduce several cardiovascular pathologic features of OSA including sympathetic hyperactivity and hypertension, are the gold-standard model to study mechanisms involved in OSA (Fletcher et al., 1992; Peng et al., 2003, 2011; Iturriaga et al., 2005, 2009; Prabhakar et al., 2005; Schulz et al., 2008; Dematteis et al., 2009; Del Rio et al., 2010, 2011a, 2012; Dumitrascu et al., 2013).

OSA produces sympathetic hyperactivity, demonstrated by an increased muscle sympathetic neural activity to blood vessels (Carlson et al., 1993) and excessive accumulation of urinary catecholamines (Dimsdale et al., 1995). Similarly, animals exposed to $\mathrm{CIH}$ show enhanced sympathetic responses to hypoxia, and develop systemic hypertension (Fletcher et al., 1992; Greenberg et al., 1999; Dick et al., 2007; Feng et al., 2008; Huang et al., 2009;
Zoccal et al., 2009; Del Rio et al., 2010; Marcus et al., 2010). The autonomic dysfunction is characterized by enhanced sympathetic outflow, a reduction of the efficiency of the cardiac baroreflex sensitivity and alterations of heart rate variability (HRV). Indeed, non-invasive spectral analysis of HRV shows an increased ratio of low (LF) to high frequency (HF) band power, with a relative predominance of the LF band and a reduced contribution of the $\mathrm{HF}$ band, suggesting preponderance of the sympathetic drive in patients with OSA (Narkiewicz et al., 1998a; Shiomi et al., 1996) and animals exposed to CIH (Lai et al., 2006; Rey et al., 2008; Del Rio et al., 2010). Furthermore, it has been shown that CIH elicits vagal withdrawal, attributed in part to neuronal loss in ambiguous nucleus (Yan et al., 2008). Therefore, it is likely that the enhanced sympathetic to parasympathetic balance along with the reduction of the baroreflex could contribute to impair HRV and the regulation of vasomotor tone of blood vessels finally eliciting systemic hypertension.

In addition, OSA syndrome is also associated with endothelial dysfunction and vascular remodeling (Ip et al., 2004; Patt et al., 2010). OSA patients show an increased intima-media thickness (Minoguchi et al., 2005; Monneret et al., 2012) and a reduced nitric oxide-mediated vasodilatation (Kato et al., 2000). Similarly, some studies found that $\mathrm{CIH}$ reduced acetylcholine (ACh)-mediated vasodilation in rats (Tahawi et al., 2001; Dopp et al., 2011), but other reported a normal endothelial function in hypertensive CIH-treated rats (Julien et al., 2003; Lefebvre et al., 2006). Indeed, Lefebvre et al. (2006) found that CIH had no effect on the ACh-mediated vasodilatation of carotid, aortic and mesenteric beds, as well as on the contractile responses induced by noradrenaline and angiotensin II (Ang II) in arteries from CIH-rats compared to the arteries from control rats. However, they found that the contraction induced by endothelin1 (ET-1) was higher in arteries from CIH-rats. More recently, Philippi et al. (2010) studied the time-course of the alteration of the endothelium dependent vasodilation in rats exposed to $\mathrm{CIH}$. They found that $\mathrm{CIH}$ produces functional and structural changes in skeletal muscle arteries within the first 2 weeks of $\mathrm{CIH}$, and those alterations were accompanied by systemic oxidative stress. Friedman et al. (2014) found that ROS generation during $\mathrm{CIH}$ activates NFATc3, which in turn increase the vascular response to ET-1. The administration of Tempol, a superoxide dismutase (SOD) mimetic, during $\mathrm{CIH}$ prevents the increased NFATc3 activity in the arteries from $\mathrm{CIH}$-exposed mice, supporting that ROS is an important upstream signal in the $\mathrm{CIH}$-induced NFATc3. Together, the available information suggest that vascular beds are affected by exposure to $\mathrm{CIH}$, and that enhanced contractile responsiveness to vasoactive molecules such as ET-1 is critically dependent on ROS formation.

\section{INTERMITTENT HYPOXIA ENHANCES CB CHEMOSENSORY DISCHARGES IN NORMOXIA AND HYPOXIA}

Patients recently diagnosed with OSA, present potentiated pressor and ventilatory responses to hypoxia (Narkiewicz et al., 1998a,b, 1999), suggesting that the peripheral hypoxic chemoreflex were enhanced by CIH. Fletcher et al. (1992) were the first to obtain evidences that the $\mathrm{CB}$ is involved in the hypertension induced by $\mathrm{CIH}$. They found that the bilateral $\mathrm{CB}$ 
denervation prevented the development of hypertension in rats exposed to $\mathrm{CIH}$ for 35 days. Despite this seminal observation, the proposal that the $\mathrm{CB}$ contributes to the progression of the cardiovascular pathologies associated to OSA was not seriously considered. However, in the last decade a growing body of new evidences have support the proposal that the $\mathrm{CB}$ contributes to the progression of the $\mathrm{CIH}$-induced hypertension (See for reviews: Prabhakar et al., 2005; Smith and Pacchia, 2007; Weiss et al., 2007; Somers et al., 2008; Garvey et al., 2009; Iturriaga et al., 2009; Dempsey et al., 2010). Recordings of rat and cat CB chemosensory discharges in situ and in vitro have demonstrate that $\mathrm{CIH}$ selectively increases basal chemosensory discharges in normoxia, and potentiates chemosensory and ventilatory responses to acute hypoxia (Peng et al., 2003, 2004; Rey et al., 2004, 2006; Prabhakar et al., 2005; Iturriaga et al., 2009; Del Rio et al., 2010, 2012). In addition, $\mathrm{CIH}$ induces plasticity of the $\mathrm{CB}$ chemosensory activity manifested as long-term facilitation. Indeed, Peng et al. (2003) found that chemosensory baseline discharges increased when the $\mathrm{CB}$ was excited by repetitive acute intermittent hypoxia in rats exposed to $\mathrm{CIH}$. They reported that following 10 episodes of $12 \% \mathrm{O}_{2}$ lasting for $15 \mathrm{~s}$, interspersed with $5 \mathrm{~min}$ of $95 \% \mathrm{O}_{2}$, the baseline chemosensory discharge increased with each episode of hypoxia, which persist for $60 \mathrm{~min}$ following the end of the hypoxic stimulus.

The mechanisms underlying the enhanced $\mathrm{CB}$ chemosensory reactivity to hypoxia induced by $\mathrm{CIH}$ are not entirely known (Iturriaga et al., 2009). Oxidative stress (Peng et al., 2003, 2009; Del Rio et al., 2010, 2012; Marcus et al., 2010), ET-1 (Rey et al., 2006, 2007; Pawar et al., 2009), Ang II (Lam et al., 2008, 2012; Fung, 2014), and pro-inflammatory cytokines (Iturriaga et al., 2009; Del Rio et al., 2011a, 2012; Lam et al., 2012) have been associated with the $\mathrm{CB}$ chemosensory potentiation. However, the primary molecular target responsible for the increased chemoreceptor discharge remains unknown. Recently, we studied the effects evoked by CIH on TASK $\mathrm{K}^{+}$channel activity and the depolarization induced by acute hypoxia in $\mathrm{CB}$ glomus cells from adult rats exposed to $\mathrm{CIH}$ (Ortiz et al., 2013). We measured membrane potential, single channel and macroscopic currents in the presence of TEA and 4-aminopyridine in CB chemoreceptor cells isolated from adult rats exposed to $\mathrm{CIH}$ for 7 days. $\mathrm{CIH}$ treatment did not change the resting membrane potential, but the hypoxicevoked depolarization increased by 2 -fold. Moreover, the hypoxic inhibition of the open probability of the TASK-K ${ }^{+}$channel was larger and faster in glomus cells from $\mathrm{CIH}$-treated rats. This novel effect of $\mathrm{CIH}$ may contribute to explain the potentiation of $\mathrm{CB}$ oxygen chemoreception.

\section{MOLECULAR MECHANISMS UNDERLYING ENHANCED CAROTID BODY CHEMOSENSORY ACTIVITY DURING INTERMITTENT HYPOXIA

ROS and reactive nitrogen species (RNS) have been proposed as mediators of the cardiovascular alterations in OSA patients (Christou et al., 2003; Lavie, 2003; Gozal and Kheirandish-Gozal, 2008; Jelic et al., 2008; Lévy et al., 2008) and animal exposed to CIH (Peng et al., 2003, 2009, 2011; Chen et al., 2005; Troncoso
Brindeiro et al., 2007; Huang et al., 2009; Del Rio et al., 2010, 2012). Studies performed in OSA patients and animals exposed to $\mathrm{CIH}$ have shown that hypoxia-reoxygenation produces systemic oxidative stress due to the accumulation of ROS and RNS. Peng et al. (2003) proposed that superoxide radical participates in the potentiation of the rat $\mathrm{CB}$ chemosensory responses to hypoxia induced by $\mathrm{CIH}$. They found that pre-treatment of rats for 10 days before and concomitant with the exposure to $\mathrm{CIH}$ with manganese (III) tetrakis (1-methyl-4-pyridyl) porphyrin pentachloride (MnTMPyP), a SOD mimetic, prevents the $\mathrm{CB}$ chemosensory potentiation. In addition, they found that $\mathrm{CIH}$ decreases the activity of the aconitase enzyme in the $\mathrm{CB}$ and the activity of the complex I of the mitochondrial electron transport chain, suggesting that the mitochondria function is affected by $\mathrm{CIH}$ and represent a potential source of ROS production (Peng et al., 2003). In addition, Peng et al. (2009) found that acute hypoxia produced a larger increase in NOX activity in CBs from rats exposed to $\mathrm{CIH}$ for 10 days compared to the NOX activity found in control CBs, suggesting that NADPH oxidase contributes to generate ROS during CIH. Recently, Schulz et al. (2014) have shown that NADPH oxidase 2 (NOX2) knockout blocks the development of the hypertension induced by $\mathrm{CIH}$. Indeed, they found that mice showed significant arterial blood pressure elevations after $\mathrm{CIH}$. The hypertension was attenuated by 1 inhibition of NOX by apocynin, whereas NOX2 was not upregulated in the heart, aorta, and femoral and carotid arteries of CIH-mice. Therefore, they suggested that the CIH-induced arterial hypertension is mediated by ROS derived from an activation of NOX2 within cells located outside the cardiovascular system.

We studied the role of nitro-oxidative stress on the enhanced $\mathrm{CB}$ chemosensory function and hypertension in rats exposed to $\mathrm{CIH}$ for 21 days (Del Rio et al., 2010). We measured 3nitrotyrosine (3-NT) formation in the $\mathrm{CB}$ as an index of oxidative stress. Superoxide reacts with NO to generate peroxynitrite, a powerful oxidizing agent that nitrates protein tyrosine-residues forming 3-NT. We found that $\mathrm{CIH}$ increased plasma lipid peroxidation and the formation of 3-NT in the CB. In addition, $\mathrm{CIH}$ enhanced the $\mathrm{CB}$ chemosensory and ventilatory responses to acute hypoxia, alters HRV and elicits hypertension. Concomitant administration of ascorbic acid reduced the increased systemic and local $\mathrm{CB}$ nitro-oxidative stress, the potentiation of $\mathrm{CB}$ chemosensory and ventilatory responses to hypoxia, as well as the hypertension in rats exposed to CIH (Del Rio et al., 2010). These results agree and extend previous observations that antioxidant treatment prevented the $\mathrm{CB}$ chemosensory potentiation (Peng et al., 2003) and the hypertension (Troncoso Brindeiro et al., 2007) in rats exposed to CIH.

The available evidence indicates that oxidative stress is involved in the $\mathrm{CIH}$-induced $\mathrm{CB}$ potentiation, but it is matter of debate whether ROS are the primary signal, because ROS per se do not increase the $\mathrm{CB}$ chemosensory discharges. Indeed, $\mathrm{H}_{2} \mathrm{O}_{2}$ does not increase the carotid chemosensory discharge in rats (Peng et al., 2009) or cats CB (Osanai et al., 1997). In addition, modification of ROS production in rat glomus cells did not alter the catecholamine secretion, suggesting a lack of a causal link between ROS and glomus cells excitability (Gonzalez et al., 2007). 


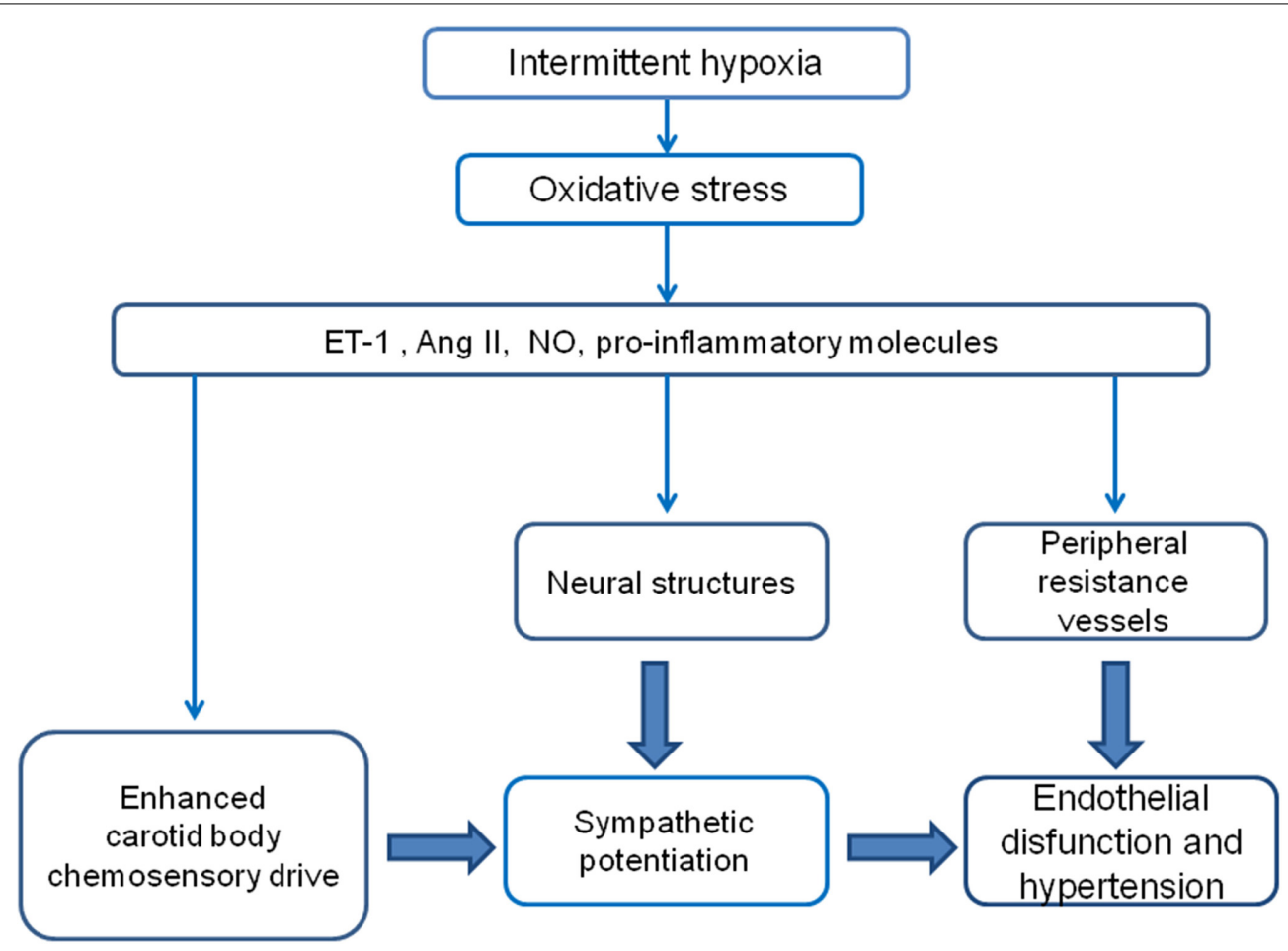

FIGURE 1 | Diagram of the proposed hypothetical mechanisms involved in the potentiation of the $\mathrm{CB}$ chemosensory response to hypoxia and the development of hypertension induced by $\mathbf{C l H}$. It is likely that the hypoxic-reoxygenation cycles enhance the $\mathrm{CB}$ chemosensitivity to hypoxia, which in turn contributes to elicit a persistent augmented sympathetic neural drive.
Thus, it is possible that other molecules activated by the oxidative stress mediate the enhancing effects of $\mathrm{CIH}$ on $\mathrm{CB}$ oxygen chemoreception (See Figure 1 and Table 1).

\section{ENDOTHELIN-1}

We and other have proposed that ET-1 is involved in the potentiation of the $\mathrm{CB}$ chemosensory discharge induced by $\mathrm{CIH}$ (Rey et al., 2006, 2007; Pawar et al., 2009; Iturriaga, 2013; Peng et al., 2013) and in the development of hypertension (Troncoso Brindeiro et al., 2007; Allahdadi et al., 2008). Rey et al. (2006) found that $\mathrm{CIH}$ increased 10-times the ET-1 immunoreactivity in endothelial, smooth muscle and glomus cells from CBs from cats exposed to $\mathrm{CIH}$ for 4 days, without changes in ET-1 plasma concentration. ET-1 elicits chemosensory excitation in both in situ and in vitro perfused cat $\mathrm{CB}$ preparation, but not in the superfused $\mathrm{CB}$ preparation, showing a predominant vascular effect. The $\mathrm{CIH}$-induced potentiation of baseline discharges and hypoxic chemosensory responses in the perfused cat $\mathrm{CB}$ preparation was reduced by the unspecific ET-1 receptor blocker bosentan (Rey et al., 2006). These results suggest that a local increase of ET1 in the $\mathrm{CB}$ may contribute to enhance the $\mathrm{CB}$ chemosensory tone induced by $\mathrm{CIH}$, through a predominant vasomotor mechanism. Pawar et al. (2009) found that CIH enhanced the basal release of ET-1 and produces upregulation of the ET-A receptor, while the administration of MnTMPyP, which prevent the oxidative stress, reduced the increased release of ET-1 and the enhanced $\mathrm{CB}$ chemosensory responses to hypoxia. In the same way, the concurrent treatment with the ET-A receptor inhibitor BQ-123
Table 1 | Possible mediator of the ClH effects on CB chemosensory potentiation.

\begin{tabular}{ll}
\hline Mediator & References \\
\hline Endothelin 1 & Rey et al., 2006, 2007; Iturriaga, 2013 \\
Endothelin-1 (dependent on ROS) & Pawar et al., 2009; Peng et al., 2013 \\
Reduced NO production (reduced & Marcus et al., 2010; Del Rio et al., \\
nNOS and eNOS-ir levels). & 2011a; Moya et al., 2012 \\
Angiotensin II (dependent on & Lam et al., 2008, 2012; Marcus et al., \\
O $_{2}$ - production signaling through & 2010; Peng et al., 2011; Fung, 2014 \\
AT1 receptor) & \\
Pro-inflammatory cytokines & Iturriaga et al., 2009; Del Rio et al., \\
& 2011b, 2012; Lam et al., 2012
\end{tabular}

prevented the development of the hypertension in rats exposed to $\mathrm{CIH}$ for 14 days (Allahdadi et al., 2008). Thus, ET-1 seems to be involved in the enhanced hypoxic $\mathrm{CB}$ chemosensory responses and in the progression of the hypertension following CIH. More recently, Peng et al. (2013) found that $\mathrm{CIH}$ increased the activity of the endothelin converting enzyme (ECE), which paralleled the raise of the ET-1 level in the neonatal rat CB. Since MnTMPyP prevented these effects, they proposed that oxidative stress was involved in the increased ET-1 expression. In addition, they found that hypoxia facilitates ET-1 release from $\mathrm{CIH}$-treated $\mathrm{CB}$, but not from control rat $\mathrm{CB}$. These results support that a ROSdependent release of ET-1, which activates the ET-A receptor is involved in the potentiation of the $\mathrm{CB}$ chemosensory responses 
to hypoxia elicited by $\mathrm{CIH}$ in neonatal rats. However, it is worth to note that Del Rio et al. (2011a) and Lam et al. (2006) found that $\mathrm{CIH}$ transiently increases the levels of ET-1 in the adult rat $\mathrm{CB}$ during the first week of $\mathrm{CIH}$, but later ET-1 levels returned to the control levels, suggesting that ET-1 may contribute to the enhanced $\mathrm{CB}$ responsiveness to hypoxia in the early phase of $\mathrm{CIH}$.

\section{NITRIC OXIDE}

We studied the changes in the expression of eNOS in the CB, along with the progression of potentiated $\mathrm{CB}$ chemosensory responses to hypoxia in rats exposed to $\mathrm{CIH}$ for 7 to 21 days (Del Rio et al., 2011a). Exposure to CIH for 7 days enhanced $\mathrm{CB}$ chemosensory responses to hypoxia and produced a significant decrease in the eNOS immunoreactivity in the $C B$, which persisted for 21 days of $\mathrm{CIH}$, suggesting that $\mathrm{CIH}$ may decrease the NO levels in the CB. Thus, we measured NO production - via nitrite generation in the incubation mediumfrom rat $\mathrm{CBs}$ exposed to $\mathrm{CIH}$, and found a reduction in the NO production after 7 days of $\mathrm{CIH}$ that correlates with the reduced eNOS expression (Del Rio et al., 2011a; Moya et al., 2012). Since NO is an inhibitory modulator of CB chemosensory discharges, we hypothesized that a reduced NO level may contribute to enhance the basal CB discharges and the chemosensory responses to hypoxia (Moya et al., 2012). This interpretation is supported by the finding of Marcus et al. (2010), showing that $\mathrm{CIH}$ decreased the expression of the nNOS in the rat $\mathrm{CB}$, suggesting that the removal of the normal inhibitory NO influence contributes to enhancing the CB chemosensory responses to hypoxia. We found a marked increase of 3-NT in the $\mathrm{CB}$ from rats exposed to $\mathrm{CIH}$, which correlates with the enhanced chemosensory responses to hypoxia (Del Rio et al., 2011a), supporting the idea that oxidative-nitrosative stress plays a critical role in $\mathrm{CB}$ chemosensory potentiation induced by $\mathrm{CIH}$ (Iturriaga et al., 2009; Del Rio et al., 2010). Thus, the available data suggests that peroxynitrite formation due to the reaction of NO with the superoxide radical is a critical step in the $\mathrm{CB}$ chemosensory potentiation induced by CIH (Del Rio et al., 2010, 2011a).

\section{ANGIOTENSIN II}

The role of Angiotensin II on the enhanced CB chemosensory responses induced by $\mathrm{CIH}$ has been extensively reviewed by Fung (2014). The CB constitutively expresses the renin-angiotensin system (RAS), and responds to Ang II due to the functional AT-1 receptor expression in the CB glomus cells (Fung et al., 2001). Lam et al. (2014) found that CIH increased the expression of angiotensinogen and AT1 receptor in the rat CB glomus cells. They also found that the elevation of intracellular $\mathrm{Ca}^{2+}$ in response to exogenous Ang II was enhanced in glomus cells from CIH-rats. The pretreatment with losartan abolished the Ang II-induced $\mathrm{Ca}^{2+}$ response, suggesting an involvement of AT1 receptors, and attenuated the levels of gp91 (phox) and macrophage infiltration in the CB. Thus, the unregulated RAS expression may play a role in the enhanced $\mathrm{CB}$ chemosensory activity and local inflammation via AT1 receptor activation during $\mathrm{CIH}$.

\section{PRO-INFLAMMATORY CYTOKINES}

Among the molecules up regulated in the $\mathrm{CB}$ by $\mathrm{CIH}$, such as ET-1, Ang II, VEGF and iNOS (Rey et al., 2006, 2007; Lam et al., 2008, 2012, 2014; Del Rio et al., 2010, 2011a,b), proinflammatory cytokines have been proposed as mediators of the CB chemosensory potentiation induced by CIH (Lam et al., 2008; Iturriaga et al., 2009; Del Rio et al., 2011a, 2012) and cardiovascular pathologies in OSA patients (Vgontzas et al., 2004; Minoguchi et al., 2005; Biltagi et al., 2008; Ryan et al., 2009). Accordingly, we studied the time-course of the changes in the immunohistological levels of TNF- $\alpha$, IL- $1 \beta$, and IL- 6 in the CB, along with the progression of the enhanced $\mathrm{CB}$ chemosensory responses to hypoxia in rats exposed to $\mathrm{CIH}$ for 7 to 21 days (Del Rio et al., 2011a). We found that $\mathrm{CIH}$ progressively increases the levels of TNF- $\alpha$ and IL- $1 \beta$ in the rat CB without modifying their plasma levels. On the contrary, Lam et al. (2012) reported that exposure of rats to intermittent hypoxia for 7 days increases the levels of IL-1 $\beta$, TNF- $\alpha$, and IL- 6 in the CB, and found macrophage infiltration, which was reduced by daily treatment with the anti-inflammatory drugs dexamethasone or ibuprofen. Oxidative stress increases the synthesis of pro-inflammatory cytokines, mediated by the activation of the transcriptional factors $N F-\kappa B$, activator protein 1 and HIF-1 $\alpha$ (Prabhakar and Semenza, 2012). In response to oxidative stress, it is known that HIF- $1 \alpha$ produced the translocation of NF- $\kappa \mathrm{B}$ to the nucleus augmenting the expression of pro-inflammatory genes such as IL- $1 \beta$, TNF- $\alpha$, and ET-1 (Reuter et al., 2010). Accordingly, we found that CBs from rats exposed to $\mathrm{CIH}$ for 21 days showed higher levels of the p65 sub-unit of NF- $\kappa \mathrm{B}$ suggesting a plausible role for this factor in the upregulation of the pro-inflammatory cytokines during $\mathrm{CIH}$ (Del Rio et al., 2012). We tested the hypothesis that CIH induced a ROSdependent increased TNF- $\alpha$ and IL- $1 \beta$ levels in the CB, which may contribute to the $\mathrm{CB}$ chemosensory potentiation (Del Rio et al., 2012). Accordingly, we studied the effects of ibuprofen on TNF- $\alpha$ and IL-1 $\beta$ levels in the rat CB, the potentiation of the $\mathrm{CB}$ chemosensory and ventilatory hypoxic responses and the development of systemic hypertension (Del Rio et al., 2012). Ibuprofen prevented the overexpression of the cytokines, the enhanced hypoxic ventilatory response and the hypertension, but failed to block the enhanced $\mathrm{CB}$ chemosensory responses. Thus, our studies suggest that the upregulation of TNF- $\alpha$ and IL- $1 \beta$ in the $\mathrm{CB}$ induced by $\mathrm{CIH}$ is linked to oxidative stress, as well as the enhanced $\mathrm{CB}$ chemosensory responsiveness to hypoxia, but the chemosensory potentiation does not depend on the increased TNF- $\alpha$ and IL- $1 \beta$ levels in the CB. However, pro-inflammatory cytokines contribute to enhance the hypoxic ventilatory response and the hypertension induced by $\mathrm{CIH}$, suggesting that multiple mechanisms may participate in the cardiorespiratory alterations induced by $\mathrm{CIH}$.

\section{CONTRIBUTION OF CENTRAL CARDIORESPIRATORY CENTERS AND ARTERIAL VESSELS TO THE HYPERTENSION INDUCED BY CIH}

The sympathetic hyperactivity induced by $\mathrm{CIH}$ is likely to be the result of the enhanced $\mathrm{CB}$ chemosensory drive, but we cannot preclude excitatory effects of $\mathrm{CIH}$ on other structures of the chemorefelex pathway. Indeed, the same molecules that are 
involved in the enhanced CB chemosensitivity (e.g., Ang II, ET-1, and $\mathrm{NO}$ ) could act at multiple sites to contribute to $\mathrm{CIH}$-induced arterial blood pressure rise (e.g., higher CNS centers, peripheral arteries vessels). The chemosensory petrosal neurons that innervate the CB glomus cells project to the NTS in the brainstem, which is the main integrative nucleus for visceral inputs. The NTS send projections to the RVLM that contain the pre-sympathetic neurons projecting to the pre-ganglionar neurons in the spinal cord. RVLM neurons participate in the control of BP, and in the CB-mediated activation of the sympathetic responses (Guyenet et al., 2010). It has been shown that $\mathrm{CIH}$ increased the expression of the neuronal activation markers $\mathrm{c}$-Fos, and FosB/ $\Delta$ Fos in the NTS and RVLM. Indeed, Greenberg et al. (1999) found that $\mathrm{CIH}$-exposure of rats for 30 days increased c-fos labeling in the NTS and the RVLM. More recently, several studies reported that $\mathrm{CIH}$ increases FosB/ $\Delta$ FosB in the subfornical organ, the median preoptic nucleus, the paraventricular nucleus, the NTS and the RVLM (Knight et al., 2011; Cunningham et al., 2012; Bathina et al., 2013). Thus, other structures outside the brainstem might contribute to intermittent hypoxia-induced hypertension (e.g., paraventricular nucleus of the hypothalamus, as shown by Sharpe et al., 2013). The available evidences strongly suggests that oxidative stress is the key mediator of the enhanced CB chemosensory responses to hypoxia and the hypertension induced by $\mathrm{CIH}$, but the actions of the oxidative stress on the $\mathrm{BP}$ regulation in rats exposed to $\mathrm{CIH}$ may occur in multiple sites of the chemoreflex pathway, including the NTS, RVLM, and/or the arterial blood vessels. Indeed, it has been proposed that superoxide anions in the brainstem contribute to elevate the arterial blood pressure in rat models of neurogenic hypertension such as the stroke-prone spontaneously hypertensive rat (Kishi et al., 2004) and Ang II induced hypertension (Chan and Chan, 2012). Although it is well known that oxidative stress, produced by Ang II and NADPH activation, in the brainstem elicits sympathetic activation, the role played by the oxidative stress induced by $\mathrm{CIH}$ in the progression of the hypertension is less known. In addition, Marcus et al. (2012) found that CIH impairs the vasodilatory responses in small arteries isolated from the skeletal muscle circulation in rats, an effect blocked by losartan, a Ang II type 1 receptor blocker. Intermittent hypoxia also caused an increase in the ratio of Ang II type 1 receptors (responsible for vasoconstriction and trophic effects) to Ang II type 2 receptors (responsible for vasodilation and anti-trophic properties) in peripheral arteries. On the other hand, oxidative stress has also been involved in the impaired vasodilatation in response to ACh in rats exposed to $\mathrm{CIH}$. Indeed, the treatment of $\mathrm{CIH}$-exposed rats with Tempol restores the normal vascular function (Phillips et al., 2006). Moreover, Dopp et al. (2011) reported that concomitant treatment with allopurinol, a xanthine oxidase inhibitor, attenuated the impairment of ACh induced vasodilatation in gracillis arteries of rats exposed to $\mathrm{CIH}$ for 14 days.

\section{CONCLUSIONS AND FUTURE DIRECTIONS}

The pathophysiological mechanisms involved in the development of hypertension in OSA are not fully understood. It is widely accepted that the $\mathrm{CIH}$-induced oxidative stress contributes to enhance the $\mathrm{CB}$ chemosensory reactivity to oxygen and to the progression of the hypertension (Figure 1). Several studies have shown that concomitant administration of antioxidants, SOD mimetic, anti-inflammatory agents, ETA, and AT-1 receptor blockers, all of them reducing the levels of ROS formation and/or blocking the downstream signaling pathways induced by $\mathrm{CIH}$, effectively prevents the enhanced CB chemosensory as well as the development of the hypertension. In addition, results showing that ablation of the CBs before the exposure to $\mathrm{CIH}$ significantly prevent the development of the hypertension strongly suggest a main role of the $\mathrm{CB}$ in the progression of the hypertension following $\mathrm{CIH}$. However, the effect of the oxidative stress on the arterial blood pressure in rats exposed to $\mathrm{CIH}$ may also occur in multiple sites of the chemoreflex pathway, including the $\mathrm{CB}$, the central cardiorespiratory centers and/or the arterial vessels. Thus, understanding how the oxidative stress and the molecules activated by $\mathrm{CIH}$ may interact at the $\mathrm{CB}$ and systemic levels would provide insights into the generation of the cardiovascular complications of OSA.

\section{ACKNOWLEDGMENTS}

This work was supported by grant 1100405 from the National Fund for Scientific and Technological Development of Chile (FONDECYT) and Project Puente/28 PUC.

\section{REFERENCES}

Abdala, A. P., McBryde, F. D., Marina, N., Hendy, E. B., Engelman, Z. J., Fudim, M., et al. (2012). Hypertension is critically dependent on the carotid body input in the spontaneously hypertensive rat. J. Physiol. 590, 4269-4277. doi: 10.1113/jphysiol.2012.237800

Allahdadi, K. J., Cherng, T. W., Pai, H., Silva, A. Q., Walker, B. R., Nelin, L. D., et al. (2008). Endothelin type A receptor antagonist normalizes blood pressure in rats exposed to eucapnic intermittent hypoxia. Am. J. Physiol. Heart Circ. Physiol. 295, H434-H440. doi: 10.1152/ajpheart.91477.2007

Arnardottir, E. S., Mackiewicz, M., Gislason, T., Teff, K. L., and Pack, A. I. (2009). Molecular signatures of obstructive sleep apnea in adults: a review and perspective. Sleep 32, 447-470.

Bathina, C. S., Rajulapati, A., Franzke, M., Yamamoto, K., Cunningham, J. T., and Mifflin, S. (2013). Knockdown of tyrosine hydroxylase in the nucleus of the solitary tract reduces elevated blood pressure during chronic intermittent hypoxia. Am. J. Physiol. Regul. Integr. Comp. Physiol. 305, R1031-R1039. doi: 10.1152/ ajpregu.00260.2013

Beebe, D. W., and Gozal, D. (2002). Obstructive sleep apnea and the prefrontal cortex: towards a comprehensive model linking nocturnal upper airway obstruction to daytime cognitive and behavioral deficits. J. Sleep Res. 11, 1-16. doi: 10.1046/j.1365-2869.2002.00289.x

Biltagi, M. A., Maguid, M. A., Ghafar, M. A., and Farid, E. (2008). Correlation of 8isoprostane, interleukin-6 and cardiac functions with clinical score in childhood obstructive sleep apnoea. Acta Paediatr. 97, 1397-1405. doi: 10.1111/j.16512227.2008.00927.x

Calhoun, D. A. (2010). Obstructive sleep apnea and hypertension. Curr. Hypertens. Rep. 12, 189-195. doi: 10.1007/s11906-010-0112-8

Carlson, J. T., Hedner, J., Elam, M., Ejnell, H., Sellgren, J., and Wallin, B. G. (1993). Augmented resting sympathetic activity in awake patients with obstructive sleep apnea. Chest 103, 1763-1768. doi: 10.1378/chest.103. 6.1763

Chan, S. H. H., and Chan, J. Y. H. (2012). Brain stem oxidative stress and its associated signaling in the regulation of sympathetic vasomotor tone. J. Appl. Physiol. 113, 1921-1928. doi: 10.1152/japplphysiol.00610.2012

Chen, L., Einbinder, E., Zhang, Q., Hasday, J., Balke, C. W., and Scharf, S. M. (2005). Oxidative stress and left ventricular function with chronic intermittent hypoxia in rats. Am. J. Respir. Crit. Care Med. 172, 915-920. doi: 10.1164/rccm.200504$5600 \mathrm{OC}$ 
Christou, K., Moulas, A. N., Pastaka, C., and Gourgoulianis, K. I. (2003). Antioxidant capacity in obstructive sleep apnea patients. Sleep Med. 4, 225-228. doi: 10.1016/S1389-9457(02)00253-8

Cunningham, J. T., Knight, W. D., Mifflin, S. W., and Nestler, E. J. (2012). An Essential role for DeltaFosB in the median preoptic nucleus in the sustained hypertensive effects of chronic intermittent hypoxia. Hypertension 60, 179-187. doi: 10.1161/HYPERTENSIONAHA.112.193789

Del Rio, R., Marcus, N. J., and Schultz, H. D. (2013). Carotid chemoreceptor ablation improves survival in heart failure: rescuing autonomic control of cardiorespiratory function. J. Am. Coll. Cardiol. 62, 2422-2430. doi: 10.1016/j.jacc.2013.07.079

Del Rio, R., Moya, E. A., and Iturriaga, R. (2010). Carotid body and cardiorespiratory alterations in intermittent hypoxia: the oxidative link. Eur. Respir. J. 36, 143-150. doi: 10.1183/09031936.00158109

Del Rio, R., Moya, E. A., and Iturriaga, R. (2011a). Differential expression of pro-inflammatory cytokines, endothelin- 1 and nitric oxide synthases in the rat carotid body exposed to intermittent hypoxia. Brain Res. 1395, 74-85. doi: 10.1016/j.brainres.2011.04.028

Del Rio, R., Moya, E. A., Parga, M. J., Madrid, C., and Iturriaga, R. (2012). Carotid body inflammation and cardiorespiratory alterations in intermittent hypoxia. Eur. Respir. J. 39, 1492-1500. doi: 10.1183/09031936.00141511

Del Rio, R., Muñoz, C., Arias, P., Court, F. A., Moya, E. A., and Iturriaga, R. (2011b). Chronic intermittent hypoxia-induced vascular enlargement and VEGF upregulation in the rat carotid body is not prevented by antioxidant treatment. Am. J. Physiol. Lung. Cell Mol. Physiol. 301, L702-L711. doi: 10.1152/ajplung.00128. 2011

Dematteis, M., Godin-Ribuot, D., Arnaud, C., Ribuot, C., Stanke-Labesque, F., Pepin, J. L., et al. (2009). Cardiovascular consequences of sleep-disordered breathing: contribution of animal models to understanding the human disease. ILAR J. 50, 262-281. doi: 10.1093/ilar.50.3.262

Dempsey, J. A., Veasey, S. C., Morgan, B. J., and O'Donnell, C. P. (2010) Pathophysiology of sleep apnea. Physiol. Rev. 90, 47-112. doi: 10.1152/physrev.00043.2008

Dick, T. E., Hsieh, Y.-H., Wang, N., and Prabhakar, N. (2007). Acute intermittent hypoxia increases both phrenic and sympathetic nerve activities in the rat. Exp. Physiol. 92, 87-97. doi: 10.1113/expphysiol.2006.035758

Dimsdale, J. E., Coy, T., Ziegler, M. G., Ancoli-Israel, S., and Clausen, J. (1995). The effect of sleep apnea on plasma and urinary catecholamines. Sleep 18, 377-381.

Dopp, J. M., Philippi, N. R., Marcus, N. J., Olson, E. B., Bird, C. E., Moran, J. J., et al. (2011). Xanthine oxidase inhibition attenuates endothelial dysfunction caused by chronic intermittent hypoxia in rats. Respiration $82,458-467$. doi: $10.1159 / 000329341$

Dumitrascu, R., Heitmann, J., Seeger, W., Weissmann, N., and Schulz, R. (2013). Obstructive sleep apnea, oxidative stress and cardiovascular disease: lessons from animal studies. Oxid. Med. Cell. Longev. 2013:234631. doi: $10.1155 / 2013 / 234631$

Eckert, D. J., and Malhotra, A. (2008). Pathophysiology of adult obstructive sleep apnea. Proc. Am. Thorac. Soc. 5, 144-153. doi: 10.1513/pats.200707-114MG

Feng, J., Chen, B. Y., and Cui, L. Y. (2008). Carotid body-mediated changes of sympathetic nerve and their relationships with hypertension. Chin. Med. J. 17, 1732-1735.

Fletcher, E. C. (2000). Effect of episodic hypoxia on sympathetic activity and blood pressure. Respir. Physiol. 119, 189-197. doi: 10.1016/S0034-5687(99)00114-0

Fletcher, E. C., Lesske, J., Behm, R., Miller, C. C. 3rd., Stauss, H., and Unger, T. (1992). Carotid chemoreceptors, systemic blood pressure, and chronic episodic hypoxia mimicking sleep apnea. J. Appl. Physiol. (1985) 72, 1978-1984.

Friedman, J. K., Nitta, C. H., Henderson, K. M., Codianni, S. J., Sanchez, L., Ramiro-Diaz, J. M., et al. (2014). Intermittent hypoxia-induced increases in reactive oxygen species activate NFATc3 increasing endothelin-1 vasoconstrictor reactivity. Vascul. Pharmacol. 60, 17-24. doi: 10.1016/j.vph.2013.11.001

Fung, M. L. (2014). The role of local renin-angiotensin system in arterial chemoreceptors in sleep-breathing disorders. Front. Physiol. 5:336. doi 10.3389/fphys.2014.00336

Fung, M. L., Lam, S. Y., Chen, Y., Dong, X., and Leung, P. S. (2001). Functional expression of angiotensin II receptors in type-I cells of the rat carotid body. Pflugers Arch. 441, 474-480. doi: 10.1007/s004240000445

Garvey, J. F., Taylor, C. T., and McNicholas, W. T. (2009). Cardiovascular disease in obstructive sleep apnoea syndrome: the role of intermittent hypoxia and inflammation. Eur. Respir. J. 33, 1195-1205. doi: 10.1183/09031936.00111208
Gonzalez, C., Agapito, M. T., Rocher, A., Gonzalez-Martin, M. C., VegaAgapito, V., Gomez-Nino, A., et al. (2007). Chemoreception in the context of the general biology of ROS. Respir. Physiol. Neurobiol. 157, 30-44. doi: 10.1016/j.resp.2007.01.016

Gonzalez, C., Almaraz, L., Obeso, A., and Rigual, R. (1994). Carotid body chemoreceptors: from natural stimuli to sensory discharges. Physiol. Rev. 74, 829-898.

Gozal, D., and Kheirandish-Gozal, L. (2008). Cardiovascular morbidity in obstructive sleep apnea: oxidative stress, inflammation, and much more. Am. J. Respir. Crit. Care Med. 177, 369-375. doi: 10.1164/rccm.200608-1190PP

Greenberg, H. E., Sica, A., Batson, D., and Scharf, S. M. (1999). Chronic intermittent hypoxia increases sympathetic responsiveness to hypoxia and hypercapnia. J. Appl. Physiol. (1985) 86, 298-305.

Guyenet, P. G., Stornetta, R. L., and Bayliss, D. A. (2010). Central respiratory chemoreception. J. Comp. Neurol. 518, 3883-3906. doi: 10.1002/cne.22435

Huang, J., Lusina, S., Xie, T., Ji, E., Xiang, S., Liu, Y., et al. (2009). Sympathetic response to chemostimulation in conscious rats exposed to chronic intermittent hypoxia. Respir. Physiol. Neurobiol. 166, 102-106. doi: 10.1016/j.resp.2009.02.010

Idiaquez, J., Santos, I., Santin, J., Del Rio, R., and Iturriaga, R. (2014). Neurobehavioral and autonomic alterations in adults with obstructive sleep apnea. Sleep Med. 15, 1319-1323. doi: 10.1016/j.sleep.2014.05.030

Ip, M. S. M., Tse, H.-F., Lam, B., Tsang, K. W. T., and Lam, W.-K. (2004) Endothelial function in obstructive sleep apnea and response to treatment. Am. J. Respir. Crit. Care Med. 169, 348-353. doi: 10.1164/rccm.200306$767 \mathrm{OC}$

Iturriaga, R. (2013). Intermittent hypoxia: endothelin-1 and hypoxic carotid body chemosensory potentiation. Exp. Physiol. 98, 1550-1551. doi: 10.1113/expphysiol.2013.075820

Iturriaga, R., and Alcayaga, J. (2004). Neurotransmission in the carotid body: transmitters and modulators between glomus cells and petrosal ganglion nerve terminals. Brain Res. Rev. 47, 46-53. doi: 10.1016/j.brainresrev.2004.05.007

Iturriaga, R., Moya, E. A., and Del Rio, R. (2009). Carotid body potentiation induced by intermittent hypoxia: implications for cardiorespiratory changes induced by sleep apnoea. Clin. Exp. Pharmacol. Physiol. 36, 1197-1204. doi: 10.1111/j.1440-1681.2009.05213.x

Iturriaga, R., Rey, S., and Del Rio, R. (2005). Cardiovascular and ventilatory acclimatization induced by chronic intermittent hypoxia: a role for the carotid body in the pathophysiology of sleep apnea. Biol. Res. 38, 335-340. doi: 10.4067/S0716-97602005000400004

Iturriaga, R., Varas, R., and Alcayaga, J. (2007). Electrical and pharmacological properties of petrosal ganglion neurons that innervate the carotid body. Respir. Physiol. Neurobiol. 157, 130-139. doi: 10.1016/j.resp.2006.12.006

Jelic, S., Padeletti, M., Kawut, S. M., Higgins, C., Canfield, S. M., Onat, D., et al. (2008). Inflammation, oxidative stress, and repair capacity of the vascular endothelium in obstructive sleep apnea. Circulation 117, 2270-2278. doi: 10.1161/CIRCULATIONAHA.107.741512

Julien, C., Bayat, S., and Levy, P. (2003). Vascular reactivity to norepinephrine and acetylcholine after chronic intermittent hypoxia in mice. Respir. Physiol. Neurobiol. 139, 21-32. doi: 10.1016/j.resp.2003.09.005

Kato, H., Nagata, K., Kawamura, S., Suzuki, A., Sato, M., Mito, Y., et al. (2000). [Vascular and metabolic reserve in a case of hypoplasia of bilateral internal carotid arteries]. Rinsho Shinkeigaku 40, 1096-1100.

Kishi, T., Hirooka, Y., Kimura, Y., Ito, K., Shimokawa, H., and Takeshita, A. (2004). Increased reactive oxygen species in rostral ventrolateral medulla contribute to neural mechanisms of hypertension in strokeprone spontaneously hypertensive rats. Circulation 109, 2357-2362. doi: 10.1161/01.CIR.0000128695.49900.12

Knight, W. D., Little, J. T., Carreno, F. R., Toney, G. M., Mifflin, S. W., and Cunningham, J. T. (2011). Chronic intermittent hypoxia increases blood pressure and expression of FosB/ $\triangle$ FosB in central autonomic regions. Am. J. Physiol. Regul. Integr. Comp. Physiol. 301, R131-139. doi: 10.1152/ajpregu.00830.2010

Koyama, Y., Coker, R. H., Stone, E. E., Lacy, D. B., Jabbour, K., Williams, P. E., et al. (2000). Evidence that carotid bodies play an important role in glucoregulation in vivo. Diabetes 49, 1434-1442. doi: 10.2337/diabetes.49.9.1434

Lai, C. J., Yang, C. C., Hsu, Y. Y., Lin, Y. N., and Kuo, T. B. (2006). Enhanced sympathetic outflow and decreased baroreflex sensitivity are associated with intermittent hypoxia-induced systemic hypertension in conscious rats. J. Appl. Physiol. (1985) 100, 1974-1982. doi: 10.1152/japplphysiol.01051.2005 
Lam, S. Y., Liu, Y., Ng, K. M., Lau, C. F., Liong, E. C., Tipoe, G. L., et al. (2012). Chronic intermittent hypoxia induces local inflammation of the rat carotid body via functional upregulation of proinflammatory cytokine pathways. Histochem. Cell Biol. 137, 303-317. doi: 10.1007/s00418-011-0900-5

Lam, S. Y., Liu, Y., Ng, K.-M., Liong, E. C., Tipoe, G. L., Leung, P. S., et al. (2014). Upregulation of a local renin-angiotensin system in the rat carotid body during chronic intermittent hypoxia. Exp. Physiol. 99, 220-231. doi: 10.1113/expphysiol.2013.074591

Lam, S. Y., Tipoe, G. L., Liong, E. C., and Fung, M. L. (2006). Hypoxia-Inducible factor (HIF)-1 $\alpha$ and Endothelin-1 expression in the rat carotid body during Intermittent hypoxia. Adv. Exp. Med. Biol. 580, 21-27. doi: 10.1007/0-38731311-7_4

Lam, S. Y., Tipoe, G. L., Liong, E. C., and Fung, M. L. (2008). Chronic hypoxia upregulates the expression and function of proinflammatory cytokines in the rat carotid body. Histochem. Cell Biol. 130, 549-559. doi: 10.1007/s00418-0080437-4

Lavie, L. (2003). Obstructive sleep apnoea syndrome-an oxidative stress disorder. Sleep Med. Rev. 7, 35-51. doi: 10.1053/smrv.2002.0261

Lefebvre, B., Godin-Ribuot, D., Joyeux-Faure, M., Caron, F., Bessard, G., Levy, P., et al. (2006). Functional assessment of vascular reactivity after chronic intermittent hypoxia in the rat. Respir. Physiol. Neurobiol. 150, 278-286. doi: 10.1016/j.resp.2005.05.020

Lévy, P., Pepin, J. L., Arnaud, C., Tamisier, R., Borel, J. C., Dematteis, M., et al. (2008). Intermittent hypoxia and sleep-disordered breathing: current concepts and perspectives. Eur. Respir. J. 32, 1082-1095. doi: 10.1183/09031936.00013308

Marcus, N. J., Li, Y. L., Bird, C. E., Schultz, H. D., and Morgan, B. J. (2010). Chronic intermittent hypoxia augments chemoreflex control of sympathetic activity: role of the angiotensin II type 1 receptor. Respir. Physiol. Neurobiol. 171, 36-45. doi: 10.1016/j.resp.2010.02.003

Marcus, N. J., Philippi, N. R., Bird, C. E., Li, Y. L., Schultz, H. D., and Morgan, B. J. (2012). Effect of AT1 receptor blockade on intermittent hypoxiainduced endothelial dysfunction. Respir. Physiol. Neurobiol. 183, 67-74. doi: 10.1016/j.resp.2012.05.025

Marin, J. M., Agusti, A., Villar, I., Forner, M., Nieto, D., Carrizo, S. J., et al. (2012). Association between treated and untreated obstructive sleep apnea and risk of hypertension. JAMA 307, 2169-2176. doi: 10.1001/jama.2012.3418

McBryde, F. D., Abdala, A. P., Hendy, E. B., Pijacka, W., Marvar, P., Moraes, D. J. A., et al. (2013). The carotid body as a putative therapeutic target for the treatment of neurogenic hypertension. Nat. Commun. 4:2395. doi: 10.1038/ncomms3395

Minoguchi, K., Yokoe, T., Tazaki, T., Minoguchi, H., Tanaka, A., Oda, N., et al. (2005). Increased carotid intima-media thickness and serum inflammatory markers in obstructive sleep apnea. Am. J. Respir. Crit. Care Med. 172, 625-630. doi: 10.1164/rccm.200412-1652OC

Monneret, D., Tamisier, R., Ducros, V., Garrel, C., Levy, P., Baguet, J. P., et al. (2012). The impact of obstructive sleep apnea on homocysteine and carotid remodeling in metabolic syndrome. Respir. Physiol. Neurobiol. 180, 298-304. doi: 10.1016/j.resp.2011.12.009

Moya, E. A., Alcayaga, J., and Iturriaga, R. (2012). NO modulation of carotid body chemoreception in health and disease. Respir. Physiol. Neurobiol. 184, 158-164. doi: 10.1016/j.resp.2012.03.019

Narkiewicz, K., Montano, N., Cogliati, C., Van De Borne, P. J., Dyken, M. E., and Somers, V. K. (1998a). Altered cardiovascular variability in obstructive sleep apnea. Circulation 98, 1071-1077. doi: 10.1161/01.CIR.98.11.1071

Narkiewicz, K., Van De Borne, P. J., Montano, N., Dyken, M. E., Phillips, B. G., and Somers, V. K. (1998b). Contribution of tonic chemoreflex activation to sympathetic activity and blood pressure in patients with obstructive sleep apnea. Circulation 97, 943-945. doi: 10.1161/01.CIR.97.10.943

Narkiewicz, K., Van De Borne, P. J., Pesek, C. A., Dyken, M. E., Montano, N., and Somers, V. K. (1999). Selective potentiation of peripheral chemoreflex sensitivity in obstructive sleep apnea. Circulation 99, 1183-1189. doi: 10.1161/01.CIR.99.9.1183

Nieto, F. J., Young, T. B., Lind, B. K., Shahar, E., Samet, J. M., Redline, S., et al. (2000). Association of sleep-disordered breathing, sleep apnea, and hypertension in a large community-based study. Sleep Heart Health Study. JAMA 283, 1829-1836. doi: 10.1001/jama.283.14.1829

Ortiz, F. C., Del Rio, R., Ebensperger, G., Reyes, V. R., Alcayaga, J., Varas, R., et al. (2013). Inhibition of rat carotid body glomus cells TASK-like channels by acute hypoxia is enhanced by chronic intermittent hypoxia. Respir. Physiol. Neurobiol. 185, 600-607. doi: 10.1016/j.resp.2012.11.015
Osanai, S., Mokashi, A., Rozanov, C., Buerk, D. G., and Lahiri, S. (1997). Potential role of $\mathrm{H}_{2} \mathrm{O}_{2}$ in chemoreception in the cat carotid body. J. Auton. Nerv. Syst. 119, 39-45. doi: 10.1016/S0165-1838(96)00129-4

Parati, G., Lombardi, C., Hedner, J., Bonsignore, M. R., Grote, L., Tkacova, R., et al. (2013). Recommendations for the management of patients with obstructive sleep apnoea and hypertension. Eur. Respir. J. 41, 523-538. doi: 10.1183/09031936.00226711

Parati, G., Lombardi, C., and Narkiewicz, K. (2007). Sleep apnea: epidemiology, pathophysiology, and relation to cardiovascular risk. Am. J. Physiol. Regul. Integr. Comp. Physiol. 293, R1671-R1683. doi: 10.1152/ajpregu.00400.2007

Pardal, R., and López-Barneo, J. (2002). Low glucose-sensing cells in the carotid body. Nat. Neurosci. 5, 197-198. doi: 10.1038/nn812

Paton, J. F. R., Sobotka, P. A., Fudim, M., Engelman, Z. J., Hart, E. C. J. McBryde, F. D., et al. (2013). The carotid body as a therapeutic target for the treatment of sympathetically mediated diseases. Hypertension 61, 5-13. doi: 10.1161/HYPERTENSIONAHA.111.00064

Patt, B. T., Jarjoura, D., Haddad, D. N., Sen, C. K., Roy, S., Flavahan, N. A., et al. (2010). Endothelial dysfunction in the microcirculation of patients with obstructive sleep apnea. Am. J. Respir. Crit. Care Med. 182, 1540-1545. doi: 10.1164/rccm.201002-0162OC

Pawar, A., Nanduri, J., Yuan, G., Khan, S. A., Wang, N., Kumar, G. K., et al. (2009). Reactive oxygen species-dependent endothelin signaling is required for augmented hypoxic sensory response of the neonatal carotid body by intermittent hypoxia. Am. J. Physiol. Regul. Integr. Comp. Physiol. 296, R735-R742. doi: 10.1152/ajpregu.90490.2008

Peng, Y. J., Nanduri, J., Raghuraman, G., Wang, N., Kumar, G. K., and Prabhakar, N. R. (2013). Role of oxidative stress-induced endothelin-converting enzyme activity in the alteration of carotid body function by chronic intermittent hypoxia. Exp. Physiol. 98, 1620-1630. doi: 10.1113/expphysiol.2013. 073700

Peng, Y.-J., Nanduri, J., Yuan, G., Wang, N., Deneris, E., Pendyala, S., et al. (2009). NADPH oxidase is required for the sensory plasticity of the carotid body by chronic intermittent hypoxia. J. Neurosci. 29, 4903-4910. doi: 10.1523/JNEUROSCI.4768-08.2009

Peng, Y. J., Overholt, J. L., Kline, D., Kumar, G. K., and Prabhakar, N. R. (2003). Induction of sensory long-term facilitation in the carotid body by intermittent hypoxia: implications for recurrent apneas. Proc. Natl. Acad. Sci. U.S.A. 100, 10073-10078. doi: 10.1073/pnas.1734109100

Peng, Y. J., Raghuraman, G., Khan, S. A., Kumar, G. K., and Prabhakar, N. R. (2011). Angiotensin II evokes sensory long-term facilitation of the carotid body via NADPH oxidase. J. Appl. Physiol. (1985) 111, 964-970. doi: 10.1152/japplphysiol.00022.2011

Peng, Y. J., Rennison, J., and Prabhakar, N. R. (2004). Intermittent hypoxia augments carotid body and ventilatory response to hypoxia in neonatal rat pups. $J$. Appl. Physiol. 97, 2020-2025. doi: 10.1152/japplphysiol.00876.2003

Peppard, P. E., Young, T., Palta, M., and Skatrud, J. (2000). Prospective study of the association between sleep-disordered breathing and hypertension. N. Engl. J. Med. 342, 1378-1384. doi: 10.1056/NEJM200005113421901

Philippi, N. R., Bird, C. E., Marcus, N. J., Olson, E. B., Chesler, N. C., and Morgan, B. J. (2010). Time course of intermittent hypoxia-induced impairments in resistance artery structure and function. Respir. Physiol. Neurobiol. 170, 157-163. doi: 10.1016/j.resp.2009.12.003

Phillips, S. A., Olson, E. B., Lombard, J. H., and Morgan, B. J. (2006). Chronic intermittent hypoxia alters NE reactivity and mechanics of skeletal muscle resistance arteries. J. Appl. Physiol. 100, 1117-1123. doi: 10.1152/japplphysiol.00994.2005

Porzionato, A., Macchi, V., and De Caro, R. (2013). Role of the carotid body in obesity-related sympathoactivation. Hypertension 61, e57. doi: 10.1161/HYPERTENSIONAHA.113.01248

Prabhakar, N. R., Peng, Y. J., Jacono, F. J., Kumar, G. K., and Dick, T. E. (2005). Cardiovascular alterations by chronic intermittent hypoxia: importance of carotid body chemoreflexes. Clin. Exp. Pharmacol. Physiol. 32, 447-449. doi: 10.1111/j.1440-1681.2005.04209.x

Prabhakar, N. R., and Semenza, G. L. (2012). Adaptive and maladaptive cardiorespiratory responses to continuous and intermittent hypoxia mediated by hypoxia-inducible factors 1 and 2. Physiol. Rev. 92, 967-1003. doi: 10.1152/physrev.00030.2011

Reuter, S., Gupta, S. C., Chaturvedi, M. M., and Aggarwal, B. B. (2010). Oxidative stress, inflammation, and cancer: How are they linked? Free Radic. Biol. Med. 49, 1603-1616. doi: 10.1016/j.freeradbiomed.2010.09.006 
Rey, S., Corthorn, J., Chacon, C., and Iturriaga, R. (2007). Expression and immunolocalization of endothelin peptides and its receptors, ETA and ETB, in the carotid body exposed to chronic intermittent hypoxia. J. Histochem. Cytochem. 55, 167-174. doi: 10.1369/jhc.6A7079.2006

Rey, S., Del Rio, R., Alcayaga, J., and Iturriaga, R. (2004). Chronic intermittent hypoxia enhances cat chemosensory and ventilatory responses to hypoxia. J. Physiol. 560, 577-586. doi: 10.1113/jphysiol.2004.072033

Rey, S., Del Rio, R., and Iturriaga, R. (2006). Contribution of endothelin-1 to the enhanced carotid body chemosensory responses induced by chronic intermittent hypoxia. Brain Res. 1086, 152-159. doi: 10.1016/j.brainres.2006.02.082

Rey, S., Tarvainen, M. P., Karjalainen, P. A., and Iturriaga, R. (2008). Dynamic timevarying analysis of heart rate and blood pressure variability in cats exposed to short-term chronic intermittent hypoxia. Am. J. Physiol. Regul. Integr. Comp. Physiol. 295, R28-R37. doi: 10.1152/ajpregu.00070.2008

Ribeiro, M. J., Sacramento, J. F., Gonzalez, C., Guarino, M. P., Monteiro, E. C., and Conde, S. V. (2013). Carotid body denervation prevents the development of insulin resistance and hypertension induced by hypercaloric diets. Diabetes 62, 2905-2916. doi: 10.2337/db12-1463

Ryan, S., Taylor, C. T., and McNicholas, W. T. (2009). Systemic inflammation: a key factor in the pathogenesis of cardiovascular complications in obstructive sleep apnoea syndrome? Thorax 64, 631-636. doi: 10.1136/thx.2008.105577

Schultz, H. D., Li, Y. L., and Ding, Y. (2007). Arterial chemoreceptors and sympathetic nerve activity: implications for hypertension and heart failure. Hypertension 50, 6-13. doi: 10.1161/HYPERTENSIONAHA.106.076083

Schulz, R., Eisele, H. J., Murzabekova, G., and Weissmann, N. (2008). [Sleep apnea and cardiovascular disease-results from animal studies]. Pneumologie 62, 18-22. doi: 10.1055/s-2007-980132

Schulz, R., Murzabekova, G., Egemnazarov, B., Kraut, S., Eisele, H. J., Dumitrascu, R., et al. (2014). Arterial hypertension in a murine model of sleep apnea: role of NADPH oxidase 2. J. Hypertens. 32, 300-305. doi: 10.1097/HJH.0000000000000016

Sharpe, A. L., Calderon, A. S., Andrade, M. A., Cunningham, J. T., Mifflin, S. W., and Toney, G. M. (2013). Chronic intermittent hypoxia increases sympathetic control of blood pressure: role of neuronal activity in the hypothalamic paraventricular nucleus. Am. J. Physiol. Heart Circ. Physiol. 305, H1772-H1780. doi: 10.1152/ajpheart.00592.2013

Shiomi, T., Guilleminault, C., Sasanabe, R., Hirota, I., Maekawa, M., and Kobayashi, T. (1996). Augmented very low frequency component of heart rate variability during obstructive sleep apnea. Sleep 19, 370-377.

Smith, M. L., and Pacchia, C. F. (2007). Sleep apnoea and hypertension: role of chemoreflexes in humans. Exp. Physiol. 92, 45-50. doi: 10.1113/expphysiol.2006.033753

Somers, V. K., White, D. P., Amin, R., Abraham, W. T., Costa, F., Culebras, A., et al. (2008). Sleep apnea and cardiovascular disease: an American Heart Association/american College Of Cardiology Foundation Scientific Statement from the American Heart Association Council for High Blood Pressure Research Professional Education Committee, Council on Clinical Cardiology, Stroke Council, and Council On Cardiovascular Nursing. In collaboration with the National Heart, Lung, and Blood Institute National Center on Sleep Disorders
Research (National Institutes of Health). Circulation 118, 1080-1111. doi: 10.1161/CIRCULATIONAHA.107.189420

Tahawi, Z., Orolinova, N., Joshua, I. G., Bader, M., and Fletcher, E. C. (2001). Altered vascular reactivity in arterioles of chronic intermittent hypoxic rats. J. Appl. Physiol. (1985) 90, 2007-2013. discussion: 2000.

Troncoso Brindeiro, C. M., Da Silva, A. Q., Allahdadi, K. J., Youngblood, V., and Kanagy, N. L. (2007). Reactive oxygen species contribute to sleep apnea-induced hypertension in rats. Am. J. Physiol. Heart Circ. Physiol. 293, H2971-H2976. doi: 10.1152/ajpheart.00219.2007

Vgontzas, A. N., Zoumakis, E., Lin, H. M., Bixler, E. O., Trakada, G., and Chrousos, G. P. (2004). Marked decrease in sleepiness in patients with sleep apnea by etanercept, a tumor necrosis factor-alpha antagonist. J. Clin. Endocrinol. Metab. 89, 4409-4413. doi: 10.1210/jc.2003-031929

Weiss, J. W., Liu, M. D., and Huang, J. (2007). Physiological basis for a causal relationship of obstructive sleep apnoea to hypertension. Exp. Physiol. 92, 21-26. doi: 10.1113/expphysiol.2006.035733

Yan, B., Soukhova-O'Hare, G. K., Li, L., Lin, Y., Gozal, D., Wead, W. B., et al. (2008). Attenuation of heart rate control and neural degeneration in nucleus ambiguus following chronic intermittent hypoxia in young adult Fischer 344 rats. Neuroscience 153, 709-720. doi: 10.1016/j.neuroscience.2008. 01.066

Young, T., Finn, L., Peppard, P. E., Szklo-Coxe, M., Austin, D., Nieto, F. J., et al. (2008). Sleep disordered breathing and mortality: eighteen-year follow-up of the Wisconsin sleep cohort. Sleep 31, 1071-1078.

Young, T., Palta, M., Dempsey, J., Skatrud, J., Weber, S., and Badr, S. (1993). The Occurrence of sleep-disordered breathing among middle-aged adults. N. Engl. J. Med. 328, 1230-1235. doi: 10.1056/NEJM199304293281704

Zoccal, D. B., Bonagamba, L. G. H., Paton, J. F. R., and Machado, B. H. (2009). Sympathetic-mediated hypertension of awake juvenile rats submitted to chronic intermittent hypoxia is not linked to baroreflex dysfunction. Exp. Physiol. 94, 972-983. doi: 10.1113/expphysiol.2009.048306

Conflict of Interest Statement: The authors declare that the research was conducted in the absence of any commercial or financial relationships that could be construed as a potential conflict of interest.

Received: 02 October 2014; accepted: 14 November 2014; published online: 02 December 2014

Citation: Iturriaga R, Andrade DC and Del Rio R (2014) Enhanced carotid body chemosensory activity and the cardiovascular alterations induced by intermittent hypoxia. Front. Physiol. 5:468. doi: 10.3389/fphys.2014.00468

This article was submitted to Integrative Physiology, a section of the journal Frontiers in Physiology.

Copyright (C) 2014 Iturriaga, Andrade and Del Rio. This is an open-access article distributed under the terms of the Creative Commons Attribution License (CC BY). The use, distribution or reproduction in other forums is permitted, provided the original author(s) or licensor are credited and that the original publication in this journal is cited, in accordance with accepted academic practice. No use, distribution or reproduction is permitted which does not comply with these terms. 